\title{
Stochastic Resonance in an Array of Dynamical Saturating Nonlinearity with Second-Order
}

\author{
Yumei Ma \\ College of Computer Science \& Technology, Qingdao University, 308 Ningxia Road, Shinan District \\ Qingdao, 266071, China \\ Lin Zhao \\ College of Automation and Electrical Engineering, Qingdao University, 308 Ningxia Road, Shinan District \\ Qingdao, 266071, China \\ Zhenkuan Pan \\ College of Computer Science \& Technology, Qingdao University, 308 Ningxia Road, Shinan District \\ Qingdao, 266071,China \\ Jinpeng Yu \\ College of Automation and Electrical Engineering, Qingdao University, 308 Ningxia Road, Shinan District \\ Qingdao,266071, China \\ E-mail:mayumei_qdu@163.com; zhaolin1585@163.com;zkpan@qdu.edu.cn;yjp1109@hotmail.com \\ www.qdu.edu.cn
}

\begin{abstract}
The transmission of weak noisy signal by parallel array of dynamical saturating nonlinearities with secondorder is studied. Firstly, the numerical results demonstrate that the output SNR can be enhanced by parallel array of dynamical saturating nonlinearities with second-order by tuning the internal noise. Secondly, the SR effects can be optimized by the self-coupling coefficient of the dynamical nonlinearity. Then, the SR effects when the nonGaussian noise acts as the external noise are superior to that with external Gaussian noise.
\end{abstract}

Keywords: stochastic resonance, dynamical nonlinearity, second-order, signal-to-noise ratio

\section{Introduction}

Stochastic resonance (SR) establishes a phenomenon where the additive noise can enhance the performance of some certain nonlinear systems [1-16]. Benzi initially observed the SR effect in climate model several decades ago [1]. Then, the existence of SR is proved with experiments by McNamara [2].Later, some new types of SR models are applied in many research fields. When
Collins designed the neuron network model, summing nonlinear units into a parallel array, the system performance can be enhanced by adjusting the coupling strength and the array noise intensity [3]. Subsequently, various SR effects as types of array SR are investigated, for instance, the Suprathreshold SR [4]. The positive role of the array noise has been found in some complex networks, e.g. stochastic pooling networks [5], scalefree networks [6] and small-world networks 
[7].Recently, the SR in some physical system focuses on the noisy bistable system, such as the bistable fractrional-order system, asymmetric bistable system and fractional harmonic oscillator and so on [8-10]. The SR effects can be measured by signal-to-noise ratio, the fisher information [11], etc.

In this paper, the SR effect in parallel array of dynamical saturating nonlinearities with second-order is firstly studied. It is demonstrated that, the SR effect occurs in arrays of dynamical saturating nonlinearities with second-order as increasing the array size and the array noise intensity. And diverse forms of the output SNR appear which against the array noise intensity and the array scale. When the Gaussian noise acts as the external noise, the self-coupling coefficient has a greater impact on the output SNR. While the self-coupling coefficient takes smaller value, the SR effect is obviously visible. With larger value of the self-coupling coefficient, the bell-shape behavior of the output is not clearly. As the array size $N \rightarrow \infty$, the output of the nonlinear systems with the external Laplacian noise precedes that with the external Gaussian noise.

\section{Theoretical Model}

We consider a weak periodic sinusoid signal $s(\mathrm{t})$ added to a white noise $\theta(\mathrm{t})$, which is independent of $s(\mathrm{t})$ with a probability density function (PDF) $f_{\theta}$ and variance $\sigma_{\theta}^{2}=E_{\theta}\left[\mathrm{x}^{2}\right]=\int_{-\infty}^{\infty} x^{2} f_{\theta}(\mathrm{x}) \mathrm{dx}$. The maximal amplitude of $s(\mathrm{t})$ is $A(|s(\mathrm{t})| \leq A)$ and the period is $T$. Next, the input mixture $\gamma(\mathrm{t})=s(\mathrm{t})+\theta(\mathrm{t})$ is applied to each identical subsystem of an uncoupled parallel array. $\alpha_{i}(\mathrm{t})$ plays a role of array noise, independent of $\gamma(\mathrm{t})$, with the same PDF and variance $\sigma_{\alpha}^{2}$.Then the output of a subsystem as

$$
\mathrm{y}_{i}(\mathrm{t})=k\left[\gamma(\mathrm{t})+\alpha_{i}(\mathrm{t})\right]
$$

Then the system output $Z(\mathrm{t})$ is written as

$$
Z(\mathrm{t})=\frac{1}{N} \sum_{i=1}^{N} y_{i}(\mathrm{t})
$$

The output SNR can be a measure of the system performance, which is defined as the power contained in the output spectral line at fundamental frequency $1 / T$ divided by the power contained in the noise background in a small frequency bin $\Delta B$ around $1 / T$, that is

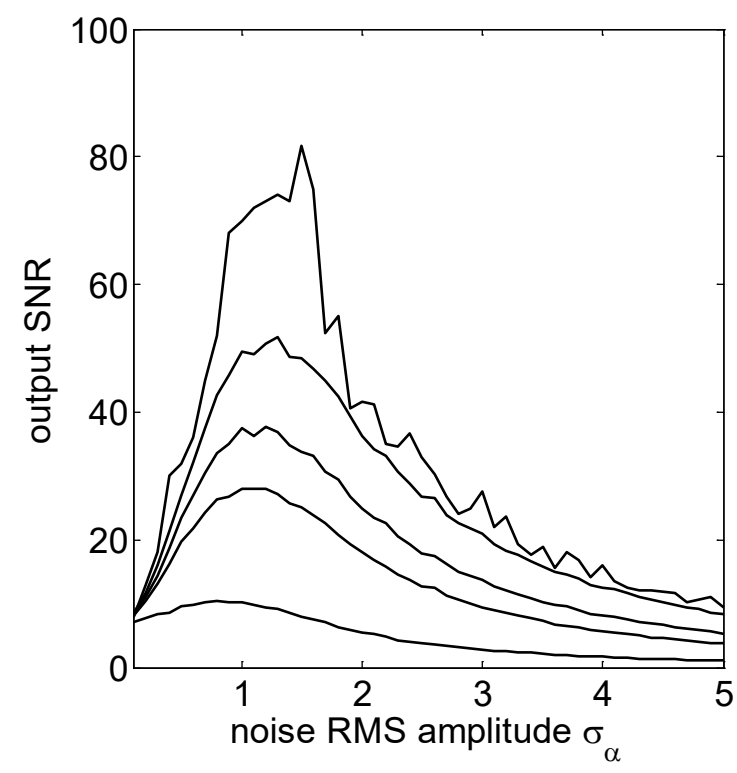

Fig. 1. Output SNR as a function of the RMS amplitude $\sigma_{\alpha}$ of the array noise $\alpha_{i}(\mathrm{t})$.

$$
R_{\text {out }}=\frac{|\langle E[\mathrm{Z}(\mathrm{t})] \exp (-i 2 \pi t / T)\rangle|^{2}}{\langle\operatorname{var}[\mathrm{Z}(\mathrm{t})]\rangle H\left(1 / T_{s}\right) \Delta B}
$$

Similarly, the input SNR is given by

$$
R_{i n}=\frac{|\langle s(\mathrm{t}) \exp (-i 2 \pi t / T)\rangle|^{2}}{\sigma_{\theta}^{2} \Delta t \Delta B}
$$

When a sinusoidal signal buried in white noise as the input signal, the input SNR is written as

$$
R_{i n}=\frac{A^{2}}{4 \sigma_{\theta}^{2} \Delta t \Delta B}
$$

When the array size $N \rightarrow \infty$, the array output SNR can be defined as

$$
R_{\text {out }}^{\infty}=\frac{\left|\left\langle E\left[\mathrm{y}_{i}(\mathrm{t})\right] \exp (-i 2 \pi t / T)\right\rangle\right|^{2}}{\left\langle E\left[\mathrm{y}_{i}(\mathrm{t}) \mathrm{y}_{j}(\mathrm{t})\right]-E^{2}\left[\mathrm{y}_{i}(\mathrm{t})\right]\right\rangle H\left(1 / T_{s}\right) \Delta B}
$$

\section{Experiment Results}

In this section, we consider a dynamical saturating nonlinearity with second-order as Eq.(7)

$\frac{d x^{2}}{d t^{2}}=-\frac{d x}{d t}-x_{i}(\mathrm{t})+C \tanh \left[\omega x_{i}(\mathrm{t})\right]+s(\mathrm{t})+\theta(\mathrm{t})+\alpha_{i}(\mathrm{t})$

where $\mathrm{C}$ is the self-coupling coefficient and $\omega$ as a slope parameter. Here, $s(\mathrm{t})=0.2 \sin (2 \pi \mathrm{t} / \mathrm{T})$ is a deterministic sinusoid with period $\mathrm{T}$. The component $\theta(\mathrm{t})$ is the external noise and it is the zero-mean generalized Gaussian noise, and some general cases 

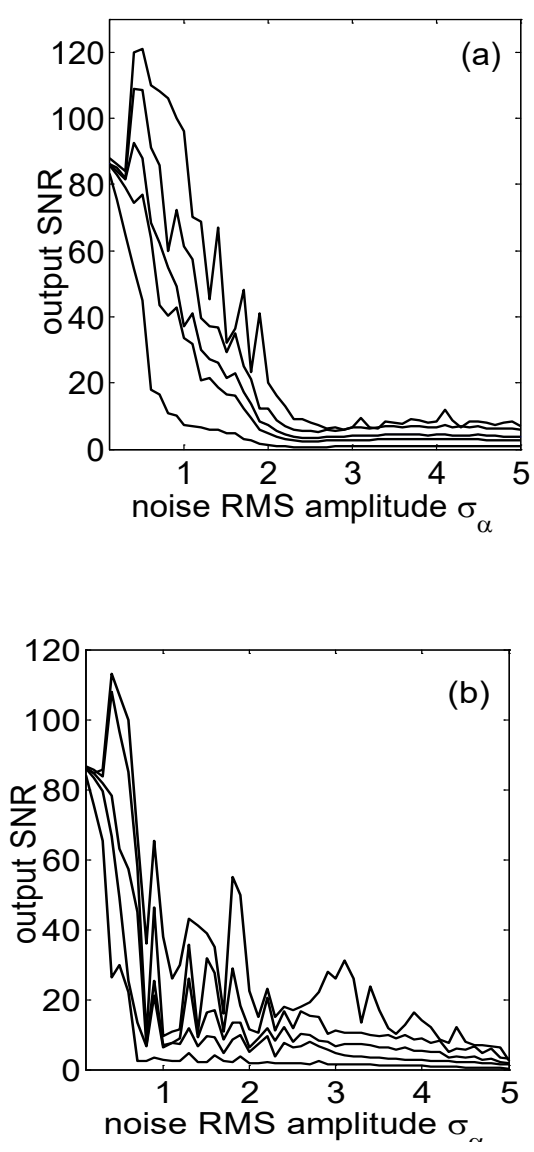

Fig. 2. Output SNR as a function of the RMS amplitude $\sigma_{\alpha}$ of the array noise $\alpha_{i}(\mathrm{t})$. The self-coupling coefficient $\mathrm{C}=1$ in (a) and $\mathrm{C}=2$ in (b).

such as Gaussian noise are contained in the class. The PDF of the generalized Gaussian noise $\theta(t)$ is defined as

$$
f_{\theta}(\mathrm{x})=\frac{c_{1}}{\sigma_{\theta}} \exp \left(-\mathrm{c}_{2}\left|\frac{x}{\sigma_{\theta}}\right|^{\mu}\right)
$$

where $c_{1}$ and $c_{2}$ is defined as Eq.(9) and Eq.(10)

$$
\begin{gathered}
\mathrm{c}_{1}=\frac{\mu}{2} \Gamma^{\frac{1}{2}}\left(3 \mu^{-1}\right) / \Gamma^{\frac{3}{2}}\left(\mu^{-1}\right) \\
\mathrm{c}_{2}=\left[\Gamma\left(3 \mu^{-1}\right) / \Gamma\left(\mu^{-1}\right)\right]^{\frac{\mu}{2}}
\end{gathered}
$$

The array noise terms $\alpha_{i}(\mathrm{t})$ are zero-mean uniformly distributed over $\left[-\sqrt{3} \sigma_{\alpha}, \sqrt{3} \sigma_{\alpha}\right]$ with RMS amplitude $\sigma_{\alpha}$.

Figure 1 and Figure 2 demonstrate the output SNR of Eq.(3)as a function of the root-mean-square (RMS) amplitude $\sigma_{\alpha}$. The slope parameter $\omega$ in Eq.(7) is set $\omega=5$. The self-coupling coefficient of the subsystem

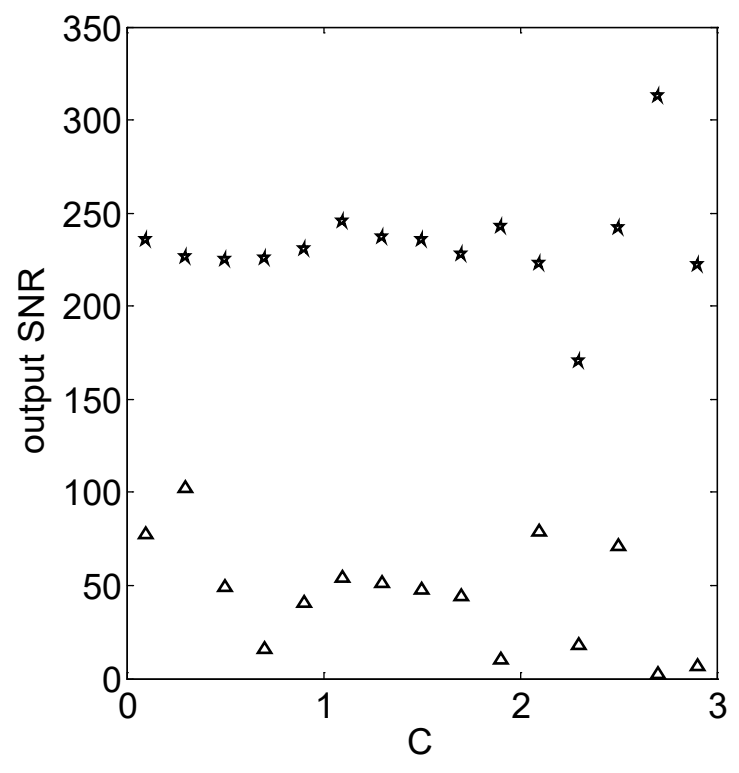

Fig. 3.Output SNR as a function of the self-coupling coefficient Cof Eq.(7).

of Eq.(7) takes $\mathrm{C}=0.5$ in Fig.1. While $\mathrm{C}=1$ in Fig.2(a) and $\mathrm{C}=2$ in Fig.2(b).In Fig.1 and Fig.2, the parameter $\mu$ of Eq.(8) is set $\mu=2$, i.e., the Gaussian noise acts as the external noise. The external noise level $\sigma_{\theta}=0.3$.

The output SNR $R_{\text {out }}$ is revealed for $\mathrm{N}=1,5,10,50$ and $\infty$ from the bottom up in Fig.1 and Fig.2.The SR effect occurs upon increasing the array noise level $\sigma_{\alpha}$ and the array size $N$. It is shown that the bell-shape behavior is obviously as the array size $N \geq 1$ in Fig.1. But the SR effect appears lightly in Fig. 2 with a certain small range of the array noise intensity when $N>1$ in Fig.2. The SR effect is more unstable while increasing the self-coupling coefficient C in Fig.2. By comparison, it is shown that the SR effect can be obtained by tuning the self-coupling coefficient $\mathrm{C}$ of the dynamical saturating nonlinearity with second-order.

Figure 3 illustrates the output SNR as a function of the self-coupling coefficient as the array size $N \rightarrow \infty$ with different external noises. The output SNR is represented by triangles when Gaussian noise acts as the external noise, while that is represented by pentagrams with external Laplacian noise. The RMS amplitude of the external noise $\sigma_{\theta}=0.3$ and the array noise intensity is $\operatorname{set} \sigma_{\alpha}=1 / \sqrt{3}$. The system output with external Laplacian noise is obviously superior to that of the nonlinear array when Gaussian noise is the external noise. 


\section{Discussion}

In this paper, SR effect is investigated firstly in an uncoupled array of dynamical saturating nonlinearities with second-order for transmitting weak noisy signal. Firstly, the SR effect occurs in parallel array of dynamical saturating nonlinearities with second-order as increasing the array size and the array noise intensity. Then, the numerical results demonstrate that higher output SNR can be obtained with smaller selfcoefficient. The output SNR with the Laplace noise as the array noise is superior to that with the Gaussian noise as the internal noise.The dynamical saturating nonlinearity with second-order can be applied in the field of physical systems actual signal processing. The results from the dynamical saturating nonlinearity with second-order may be beneficial to theoretical research and signal processing.

\section{Acknowledgements}

This work is partially supported by the National Natural Science Foundation of China (61501276, 61573204), the China Postdoctoral Science Foundation (2016M592139), the Qingdao Postdoctoral Application Research Project(2015120).

\section{References}

1. R. Benzi, G. Parisi, A. Sutera and A. Vulpiani, Stochastic resonance in climate changes, Tellus.34 (1981) 10-16.

2. McNamara B, Wiensenfeld K, Theory of stochastic resonance. Physical Review A.39 (1989) 4854-4869.

3. J.J.Collins, C. C. Chow, T.T.Imhoff, Stochastic resonance in neuron models, Physical Review E. 1995, 52: 3321-3324.

4. N. G.Stocks, Suprathreshold stochastic resonance in multilevel threshold systems, Physical Review Letters, 84 (2000) 2310-2313.
5. M.D. Mcdonnell, P. O. Amblard, N. G. Stocks, Stochastic Pooling Networks, Journal of Statistical Mechanics Theory \& Experiment. 1 (2009) P01012.

6. M. Perc, Stochastic resonance on weakly paced scale-free networks via a pacemaker, Physical Review E. E 76 (2007) 066203.

7. P. Matja, stochastic resonance on excitable small-world network via a pacemaker, Physical Review E. 76 (2007) 066203.

8. Z. Lai and Y. Leng, Weak-signal detection based on the stochastic resonance of bistable Duffing oscillator and its application in incipient fault diagnosis, Mechanical System and Signal Processing. 81 (2016) 60-74.

9. F. Guo, C.Zhu, X. Cheng and H. Li, Stochastic resonance in a fractional harmonic oscillator subject to random mass and signal-modulated noise, Physica A. 459 (2016) 86-91.

10. P. Shi, Q. Li and D. Han, Stochastic resonance in a new asymmetric bistable system driven by unrelated multiplicative and additive noise, Chinese Journal of Physics. 54 (2016) 526-532.

11. Y. Ma,F. Duan,F. Chapeau-Blondeau and D. Abbott, Weak-Periodic Stochastic Resonance in a Parallel Array of Static Nonlinearities, PLoS ONE. 8(3) 2013 e58507.

12. B. Lindner, J. Garcia and A. Neiman, Schimansky-Geier L. Effects of noise in excitable systems, Physics Reports. 392 (2004) 321-424.

13. F. Chapeau-Blondeau and X. Godivier, Theory of stochastic resonance in signal transimission by static nonlinear systems, Physical Review E. 55 (1997) 14781495.

14. B. Chen, Y. Zhu, J. Hu and M.Zhang, Weak signal detection: Condition for noise induced enhancement, Digital Signal Processing. 23(2013)1585-1591.

15. H. Droogendijk, M. J. de Boer, R. G. p. Sanders and G. J. M. Krijnen, Stochastic Resonance in a VoltageControlled Micromechanical Slider, Microelectromechanical Systems. 24(3) (2015)651-660.

16. T. Djurhuus and V. Krozer, A Generalized Model of Noise Driven Circuits with Application to Stochastic Resonance, Circuits and Systems I: Regular Papers, IEEE Transactions on .62(8) (2015)1981-1990. 